\title{
BMJ Open Association between chronic kidney disease and incident diagnosis of dementia in England: a cohort study in Clinical Practice Research Datalink
}

To cite: Hiramatsu R, Iwagami M, Nitsch D. Association between chronic kidney disease and incident diagnosis of dementia in England: a cohort study in Clinical Practice Research Datalink. BMJ Open 2020;10:e033811. doi:10.1136/ bmjopen-2019-033811

- Prepublication history and additional material for this paper are available online. To view these files, please visit the journal online (http://dx.doi org/10.1136/bmjopen-2019033811).

$\mathrm{RH}$ and $\mathrm{Ml}$ contributed equally.

Received 23 August 2019 Revised 10 January 2020 Accepted 14 April 2020

Check for updates

(C) Author(s) (or their employer(s)) 2020. Re-use permitted under CC BY-NC. No commercial re-use. See rights and permissions. Published by BMJ.

${ }^{1}$ Kidney Centre, Toranomon Hospital, Tokyo, Japan

${ }^{2}$ Department of Health Services Research, University of Tsukuba, Tsukuba, Japan

${ }^{3}$ Department of Non-

Communicable Disease

Epidemiology, London School of Hygiene and Tropical Medicine, London, UK

Correspondence to

Dr Masao Iwagami;

iwagami-tky@umin.ac.jp

\section{ABSTRACT}

Objectives To investigate the association between chronic kidney disease (CKD) and dementia diagnosis in a real-world primary care setting in England.

Design Matched cohort study.

Settings English primary care in the Clinical Practice Research Datalink.

Participants People aged $\geq 18$ years with predialysis CKD (stages 3-5, defined as two measurements of estimated glomerular filtration rate $<60 \mathrm{~mL} / \mathrm{min} / 1.73 \mathrm{~m}^{2}$ for 3 months) from 2004 to 2014, and people without known CKD who were matched on age, sex, general practice and calendar time in a 1:1 ratio.

Primary and secondary outcome measures First-ever diagnosis of dementia recorded by GPs. We also examined all-cause death as a secondary outcome to discuss potential competing risk of mortality in the association between CKD and dementia diagnosis.

Results In a matched cohort of 242349 pairs with and without CKD (mean age $75.4 \pm 9.7$ years, $39.3 \%$ male), the crude incidence rate of dementia diagnosis was 11.4/1000 and 9.4/1000 person-years, respectively. There was an association between CKD status and incident dementia diagnosis in the first 6 months of the follow-up (adjusted rate ratio (aRR) 1.58, 95\% $\mathrm{Cl} 1.44$ to 1.74 ), which attenuated after 6 months (aRR $1.12,95 \% \mathrm{Cl} 1.08$ to 1.16). Among patients with CKD, there was no evidence of association between CKD stage and incident dementia diagnosis; compared with stage $3 \mathrm{a}$, aRR $(95 \% \mathrm{Cl})$ was 1.04 (0.91 to 1.18) for stage $3 b$ and 0.94 (0.74 to 1.20) for stages 4 or 5 in the first 6 months, and 0.97 ( 0.92 to 1.01 ) and 0.89 ( 0.80 to 0.98 ) thereafter. We found a strong association between worsening CKD stage and all-cause mortality.

Conclusion We identified a co-occurrence of detection of CKD and dementia in real-world clinical practice and a strong competing risk of mortality in the association between CKD stage and dementia, while a weak association between CKD status and dementia was suggested in the long term.

\section{INTRODUCTION}

The prevalence of both chronic kidney disease (CKD) and dementia is increasing globally, especially in high-income countries with ageing populations. ${ }^{1-3}$ It is known
Strengths and limitations of this study

- We used one of the largest databases of electronic health records collected by general practitioners, which allowed us to examine the association between chronic kidney disease and dementia diagnosis a real-world primary care setting.

- Chronic kidney disease status and stage were strictly defined based on serum creatinine information, instead of diagnosis codes with limited validity.

- We adjusted for a wide range of comorbidities and lifestyle-related factors (eg, smoking status and body mass index) in the association between chronic kidney disease and dementia.

- Although the positive predictive value of dementia diagnosis is known to be high in UK primary care, its sensitivity may be low, meaning that the crude incidence rate of dementia might be underestimated.

- The lack of details to accurately classify dementia subtypes (eg, Alzheimer's disease, vascular dementia and Lewy body dementia) precluded us from analysing the relationship between chronic kidney disease and dementia subtypes.

that CKD and dementia are both associated with an increased healthcare burden. ${ }^{45} \mathrm{~A}$ UK study demonstrated that primary and secondary care resource utilisation increased after a diagnosis of dementia, ${ }^{4}$ while another UK study suggested that inpatient and outpatient healthcare costs increased, as CKD stages deteriorated. ${ }^{5}$

Both the kidneys and the brain are smallvessel organs with haemodynamic similarities, and can be damaged simultaneously. Much effort has been devoted to exploring the biological mechanisms in the association between CKD and dementia to date. ${ }^{6-10}$ First, CKD and dementia share cardiovascular risk factors including smoking, obesity and diabetes mellitus. In addition, evidence suggests that CKD-specific factors, such as anaemia and electrolyte disorders (eg, hyponatraemia), are associated with dementia. 
Moreover, poor nutrition and chronic inflammation, which are often observed in patients with CKD, could contribute to the development of dementia. ${ }^{67}$

Previous studies have reported an approximately threefold to fivefold increase in the burden of dementia in dialysis patients, compared with the general population. ${ }^{11}$ Among the non-dialysis population, CKD has been associated with cognitive dysfunction and/or dementia in cross-sectional studies. ${ }^{12} 13$ A recent Japanese longitudinal cohort study showed that CKD was associated with dementia independent of measured vascular risk factors and cerebral small-vessel disease (identified by MRI) at baseline. ${ }^{14}$ A meta-analysis of five cohort studies found a marginally significant association between decreased kidney function (estimated glomerular filtration rate (eGFR) $<60 \mathrm{~mL} / \mathrm{min} / 1.73 \mathrm{~m}^{2}$ ) and incidence of cognitive impairment or dementia (relative risk 1.28, 95\% CI 0.99 to 1.65 ), and a significant association between albuminuria and dementia (relative risk $1.35,95 \%$ CI 1.06 to 1.73). ${ }^{15}$

Given that both CKD and dementia incur a healthcare burden, it is important to understand the association between CKD status or stage and diagnosis of dementia in real-world clinical practice. However, according to a recent review of the literature, the incidence of dementia in early CKD in routine clinical care is unknown. ${ }^{8}$ Therefore, using a large contemporary dataset from UK primary care, the objectives of current study were (i) to investigate the incidence of dementia diagnoses in patients with CKD (stages 3-5, defined as two measurements of eGFR $<60 \mathrm{~mL} / \mathrm{min} / 1.73 \mathrm{~m}^{2}$ for 3 months), compared with those without known CKD, and (ii) to investigate whether there was a graded increase in the incidence of dementia diagnoses according to CKD stage (CKD stage $3 \mathrm{a}, 3 \mathrm{~b}$ and 4 or 5), with careful approach to deal with bias inherent in the real-world data (eg, by quantifying the extent of competing risk of mortality which may affect duration of follow-up for dementia diagnoses).

\section{MATERIALS AND METHODS \\ Data sources}

General practitioners (GPs) in the UK act as gatekeepers to the wider healthcare system, and almost all UK citizens are registered with GPs from whom they receive routine healthcare. GPs have been using computerised health records since the late 1980 s to record clinically relevant lifestyle information, clinical events or diagnoses, prescriptions, specialist referrals, hospital administration and immunisation for individual patients. The Clinical Practice Research Datalink (CPRD) is a database of anonymised electronic health records made available for research. At the time of this study, the CPRD contained routinely recorded data for about $7 \%$ of the UK population, and was deemed broadly representative. ${ }^{16}$ In order to gain more comprehensive information on ethnicity, we employed around $60 \%$ of CPRD data that were linked to Hospital Episodes Statistics (HES) in England. ${ }^{17}$ The data were also linked to the Index of Multiple Deprivation, which is a validated, small area-level variable capturing the socioeconomic status (SES) of study participants. ${ }^{18}$ Patients or the public were not involved in the design, conduct, reporting or dissemination of our research.

\section{Study population}

All individuals aged 18 years or older with data in the HES-linked CPRD from 1 April 2004 to 31 March 2014 were potentially eligible for inclusion, according to the following criteria: (i) 1 year after practice registration, to ensure that medical information from previous GPs had been incorporated in the electronic health records of current $\mathrm{GPs}^{19}$; (ii) the date that the practice reached CPRD quality control standards, in terms of data quality ${ }^{16}$ and (iii) after 1 April 2004, when the Quality and Outcomes Framework was introduced, enhancing kidney function tests in primary care in the UK. ${ }^{20}$ We excluded patients on renal replacement therapy (haemodialysis, peritoneal dialysis or kidney transplantation) because our research focus lay on the association between predialysis CKD and dementia.

\section{CKD cohort}

Patients with CKD at stages 3-5 were identified based on two consecutive measurement of eGFR $<60 \mathrm{~mL} /$ $\min / 1.73 \mathrm{~m}^{2},>3$ months apart. ${ }^{21}$ eGFR was calculated from serum creatinine records in CPRD using the Chronic Kidney Disease Epidemiology Collaboration equation, ${ }^{22}$ after having applied the correction factor of 0.95 to the recorded serum creatinine levels to allow for uncalibrated creatinine measurements. The patients were included in the CKD cohort on the date when their second eGFR was $<60 \mathrm{~mL} / \mathrm{min} / 1.73 \mathrm{~m}^{2}$ (index date).

\section{Matched comparison cohort (no-known CKD cohort)}

To establish a comparison group, people without known CKD were randomly selected from the rest of the study population (ie, people without known CKD in stages 3-5) (figure 1). For each patient with CKD (stages 3-5), we randomly selected a person without known CKD of the same age and sex from the same practice, at the same calendar time as the start of the follow-up. Matching based on age, sex, general practice and calendar time is justified because CKD status largely depends on age and $\operatorname{sex},{ }^{23}$ while the identification and management of CKD and dementia can vary across general practice and calendar time.

\section{Outcome}

The outcome of interest was the incident diagnosis of dementia, based on diagnosis codes (Read codes) recorded in the CPRD by GPs. ${ }^{2425}$ Dementia here includes Alzheimer's disease, vascular dementia and other types of dementia such as Lewy body dementia (a code list is shown in online supplementary table S1). In this study, we did not differentiate between dementia subtypes because (i) it is generally difficult to differentiate between them in real-world clinical practice and (ii) many patients had 


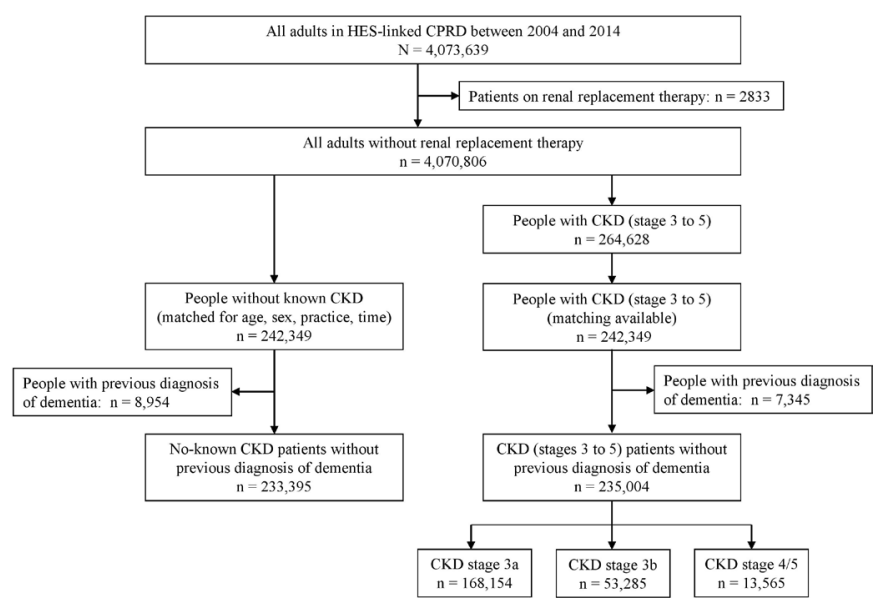

Figure 1 Flow chart of the selection of eligible patients with and without chronic kidney disease. CKD, chronic kidney disease; CPRD, Clinical Practice Research Datalink; HES, Hospital Episode Statistics.

diagnosis codes (Read codes) suggesting unspecified dementia in the current datasets. We focused on the incidence of new (ie, first-ever) diagnoses of dementia in the CPRD, and therefore excluded people with any diagnosis code of dementia recorded prior to the cohort entry in the CKD and matched comparison cohorts.

\section{Covariates}

In addition to the matched factors (ie, age, sex, general practice and calendar time), we considered ethnicity, SES, smoking status, body mass index (BMI) and comorbidities including asthma, chronic obstructive pulmonary disease (COPD), severe mental illness, depression, epilepsy, coronary artery disease, peripheral arterial disease, heart failure, stroke and transient ischaemic attack, arterial fibrillation, diabetes mellitus, hypertension, rheumatoid arthritis, hypothyroidism, osteoporosis and cancer. We

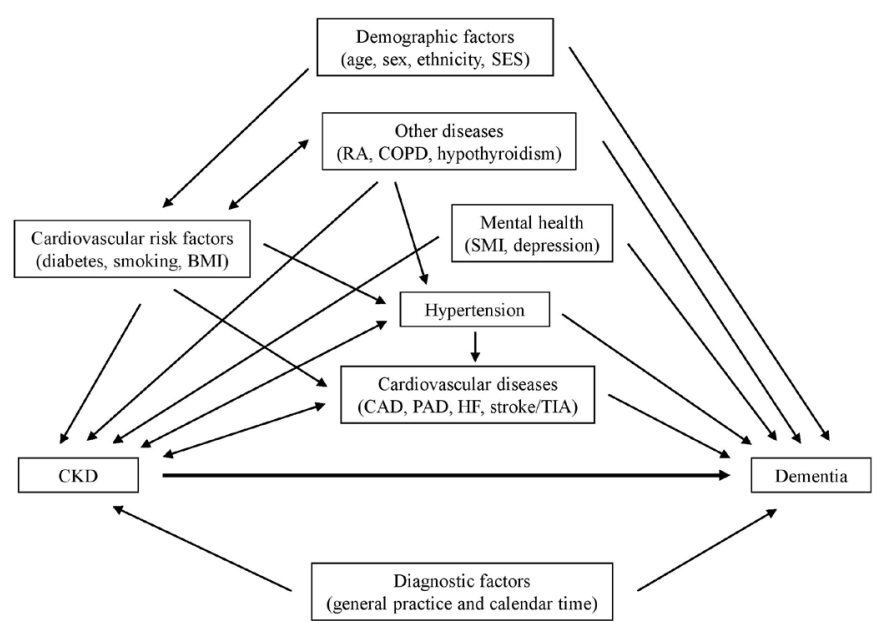

Figure 2 Causal diagram of the association between chronic kidney disease and dementia. BMI, body mass index; CAD, coronary artery disease; CKD, chronic kidney disease; COPD, chronic obstructive pulmonary disease; HF, heart failure; RA, rheumatoid arthritis; SES, socioeconomic status; SMI, severe mental illness; TIA, transient ischaemic attack. categorised ethnicity into white, South Asian, black and others/mixed based on a previously established algorithm using the CPRD and the HES, ${ }^{17}$ and grouped people with missing ethnicity information (neither in the CPRD nor the HES) into the white group, based on previous studies. ${ }^{26}$ Individual SES data were categorised into quintiles $(\mathrm{Q})$ from least deprived $(\mathrm{Q} 1)$ to most deprived $(\mathrm{Q} 5)$. For a small number of patients with missing information on individual-level SES, their SES status was replaced with the SES status of their general practice (based on their postcode). Smoking status was classified as never, current and ex-smoker, using the data recorded closest to the cohort entry. Each comorbidity was defined as present if a relevant diagnosis Read code was recorded at least once between the CPRD registration and the cohort entry. In addition, prescription records were used to identify patients with hypertension and diabetes mellitus.

\section{Statistical analysis to compare matched patients with and without CKD}

We first showed the baseline characteristics of patients in the CKD and matched comparison cohorts, with $\chi^{2}$ tests for comparison. We found that the data did not fulfil the proportional assumptions for Cox modelling, and therefore used unconditional Poisson regression models with interactions with a follow-up time to calculate stratumspecific rate ratios (RR) for incident dementia diagnosis in the first 6 months of follow-up and thereafter (ie, $>6$ months after the cohort entry).

Using prior knowledge, a causal diagram containing variables that were deemed to be associated with both CKD and dementia was created to aid the selection of potential confounders among the aforementioned covariates (figure 2). Demographic factors (age, sex, ethnicity and SES), diagnostic factors (general practice and calendar time), cardiovascular risk factors (diabetes mellitus, smoking and $\mathrm{BMI})^{27}$ and comorbidities including severe mental illness and depression, ${ }^{28}$ rheumatoid arthritis, ${ }^{29} 30$ $\mathrm{COPD}^{31}$ and hypothyroidism ${ }^{32}{ }^{33}$ were all considered to be associated with both CKD and dementia. We considered hypertension and cardiovascular diseases (coronary artery disease, peripheral arterial disease, heart failure, stroke and transient ischaemic attack) to be potentially both mediators and confounders between CKD and dementia, and therefore decided to show the results of models with adjustments for these variables as a sensitivity analysis.

Moving on from this conceptual framework, we fitted a sequence of models as follows: model 1 was minimally adjusted for matched factors (age, sex, general practice and calendar time); model 2 was also adjusted for SES, ethnicity and shared cardiovascular risk factors (smoking status, BMI and diabetes mellitus) as a complete case analysis; model 3 was additionally adjusted for comorbidities considered to be potential confounders (severe mental illness, rheumatoid arthritis, COPD, hypothyroidism and depression) and model 4 (regarded as a sensitivity 
analysis) was additionally adjusted for hypertension and cardiovascular diseases.

To assess the influence of the competing risk of allcause mortality (ie, if patients with CKD died earlier, they may be less likely to be diagnosed with dementia than patients without CKD), we also estimated stratum-specific RRs for death in the first 6 months of a follow-up and thereafter separately, adjusting for the same covariates as the Poisson regression analyses for dementia diagnosis. We did not conduct a competing risk analysis, because our aim here was to describe the incidence of dementia and mortality over time in these patients as aetiological research, rather than prognostic research that aims to predict the probability of dementia in patients with and without CKD. ${ }^{34}$

\section{Statistical analysis to compare patients with different stages of CKD in the CKD cohort}

To investigate whether there is a graded association between the severity of CKD and incident diagnosis of dementia in the CKD cohort, the kidney function of patients on the day of cohort entry was classified into three categories; eGFR $45-60 \mathrm{~mL} / \mathrm{min} / 1.73 \mathrm{~m}^{2}$ (namely, CKD stage 3a); eGFR $30-44 \mathrm{~mL} / \mathrm{min} / 1.73 \mathrm{~m}^{2}$ (namely, CKD stage $3 \mathrm{~b}$ ) and eGFR $<30 \mathrm{~mL} / \mathrm{min} / 1.73 \mathrm{~m}^{2}$ (namely, CKD stage 4 or 5$).{ }^{21}{ }^{35}$ Similar to the main analysis, the aforementioned models (models 1-4) were fitted in the first 6 months of follow-ups and thereafter separately, regarding CKD stage as exposure and dementia and allcause mortality as separate outcomes.

All statistical analyses were performed in STATA V.14.0.

\section{Patient and public involvement}

No patients were involved in the current study.

\section{RESULTS}

Among the 4070806 adults without renal replacement therapy registered in the HES-linked CPRD between April 2004 and March 2014, we identified 264628 patients with CKD (stages 3-5) (figure 1). Of these, 242349 with CKD (mean age $75.4 \pm 9.7$ years, $39.3 \%$ male) were individually matched to people without known CKD in terms of age, sex and general practice on the same calendar date for cohort entry. Then, we excluded $7345(3.0 \%)$ and 8954 (3.7\%) patients with previous diagnoses of dementia from the CKD and matched comparison cohort, respectively. Compared with patients without known CKD $(\mathrm{n}=233395)$, patients with CKD $(\mathrm{n}=235004)$ were more likely to be affluent, ex-smokers and obese (table 1). Comorbidities except for osteoporosis and epilepsy were more commonly diagnosed for patients in the CKD cohort than in the matched comparison cohort.

During the follow-up (median 3.0 years), a total of 20658 patients were newly diagnosed with dementia, including $11973(5.1 \%)$ in the CKD cohort $(n=235004)$ and $8685(3.7 \%)$ in the matched comparison cohort $(\mathrm{n}=233395)$. The crude incidence rate of dementia diagnosis was $11.4 / 1000$ and 9.4/1000 person-years in matched patients with and without CKD, respectively. Table 2 shows the adjusted RR, comparing patients with and without CKD for the incidence of dementia diagnosis and all-cause mortality. The adjusted RR (95\% CI) for the incident dementia diagnosis in model 3 was 1.58 (1.44 to 1.74 ) within the first 6 months, and 1.12 (1.08 to 1.16 ) from 6 months onwards. The competing risk of death was not strongly suggested: the adjusted RR $(95 \% \mathrm{CI})$ for allcause mortality in model 3 was 1.01 (0.97 to 1.06) and 1.12 (1.10 to 1.14 ) in the first 6 months and thereafter, respectively. Additional adjustments for hypertension and cardiovascular diseases in model 4 (as a sensitivity analysis) did not substantially change the results.

Within the CKD cohort, the crude incidence rate of dementia diagnosis was $10.7,13.8$, and 13.0/1000 person-years in patients with CKD stages $3 \mathrm{a}, 3 \mathrm{~b}$ and 4 or 5 , respectively. Similarly to the main analysis for patients with and without CKD (table 2), the RRs (comparing CKD stages $3 a, 3 b$ and $4 / 5$ ) for the incident dementia diagnosis were generally greater within the first 6 months than thereafter (table 3). Compared with patients with CKD stage 3a, the adjusted RR (95\% CI) for incident dementia diagnosis in model 3 was 1.04 (0.91 to 1.18 ) in patients with CKD stage $3 \mathrm{~b}$ and 0.94 (0.74 to 1.20 ) in patients with CKD stage $4 / 5$ within the first 6 months of follow-ups, while it was 0.97 (0.92 to 1.01) in patients with CKD stage $3 \mathrm{~b}$ and 0.89 (0.80 to 0.98 ) in patients with CKD stages $4 / 5$, from 6 months onwards. Competing risk of death (against the incidence of dementia diagnosis) was strongly suggested: compared with patients with CKD stage 3a, the adjusted RR (95\% CI) for all-cause mortality in model 3 was 1.85 (1.74 to 1.95 ) and 4.33 (4.06 to 4.61) in patients with CKD stages $3 \mathrm{~b}$ and $4 / 5$ in the first 6 months, and 1.51 (1.48 to 1.54) and 2.56 (2.47 to 2.63) after 6 months of follow-ups, respectively. Additional adjustments for hypertension and cardiovascular diseases in model 4 (as a sensitivity analysis) did not substantially change the results.

\section{DISCUSSION \\ Summary of findings}

In this study based on a large English population-based primary care cohort, CKD (stages 3-5) was associated with incident diagnoses of dementia, after adjusting for a wide range of comorbidities. The association was not constant over time; dementia was diagnosed at a higher rate among patients with CKD soon after they were identified as having CKD, and the association was weaker after 6 months. However, there was still a statistically significant association between CKD status and dementia (the adjusted RR 1.12 (95\% CI 1.08 to 1.16) from 6 months onwards). There was no graded association between CKD stage and the incidence of dementia diagnosis, while there was a strong association between CKD stage and mortality. 
Table 1 Baseline characteristics of matched patients with and without CKD (stages 3-5) by CKD status and stage

\begin{tabular}{|c|c|c|c|c|c|c|}
\hline \multirow[b]{3}{*}{ Variables } & \multicolumn{3}{|c|}{ By CKD status ( $n=468399)$} & \multicolumn{3}{|c|}{ By CKD stage $(n=235004)$} \\
\hline & $\begin{array}{l}\text { Matched } \\
\text { patients } \\
\text { without known } \\
\text { CKD } \\
(n=233395)\end{array}$ & $\begin{array}{l}\text { Matched } \\
\text { patients with } \\
\text { CKD } \\
(n=235004)\end{array}$ & P value & $\begin{array}{l}\text { CKD stage 3a } \\
(n=168154)\end{array}$ & $\begin{array}{l}\text { CKD stage 3b } \\
(n=53285)\end{array}$ & $\begin{array}{l}\text { CKD stage } 4 / 5 \\
(n=13565)\end{array}$ \\
\hline & $\mathbf{N}(\%)$ & $\mathbf{N}(\%)$ & & N (\%) & N (\%) & $\mathbf{N}(\%)$ \\
\hline Sex (male) & 93050 (39.9) & $93233(39.7)$ & 0.173 & $67470(40.1)$ & 19915 (37.4) & $5848(43.1)$ \\
\hline Ethnicity & & & $<0.001$ & & & \\
\hline White/Non-recorded & $229666(98.4)$ & 230906 (98.3) & & 165254 (98.3) & $52439(98.4)$ & $13213(97.4)$ \\
\hline South Asian & $1772(0.8)$ & $2260(1.0)$ & & $1625(1.0)$ & $464(0.9)$ & $171(1.3)$ \\
\hline Black & $1119(0.5)$ & $1031(0.4)$ & & $710(0.4)$ & $200(0.4)$ & $121(0.9)$ \\
\hline Q2 & $59494(25.5)$ & $58693(25.0)$ & & $42482(25.3)$ & $13081(24.6)$ & $3130(23.1)$ \\
\hline Q3 & $48486(20.8)$ & 49038 (20.9) & & 34908 (20.8) & $11202(21.0)$ & $2928(21.6)$ \\
\hline Q4 & $40785(17.5)$ & $43396(18.5)$ & & $30561(18.2)$ & $10104(19.0)$ & $2731(20.1)$ \\
\hline Q5 (most deprived) & 30024 (12.9) & 32475 (13.8) & & 22335 (13.3) & 7949 (14.9) & 2191 (16.2) \\
\hline Smoking status & & & $<0.001$ & & & \\
\hline Non-smoker & 87595 (37.5) & 77333 (32.9) & & $54547(32.4)$ & $18137(34.0)$ & 4649 (34.3) \\
\hline Current smoker & 35633 (15.3) & $28623(12.2)$ & & $20212(12.0)$ & 6609 (12.4) & 1802 (13.3) \\
\hline Ex-smoker & $104637(44.8)$ & $128310(54.6)$ & & $93074(55.4)$ & $28283(53.1)$ & 6953 (51.3) \\
\hline Missing & 26295 (11.3) & $14597(6.2)$ & & $8053(4.8)$ & $4750(8.9)$ & 1794 (13.2) \\
\hline \multicolumn{7}{|l|}{ Comorbidities } \\
\hline Asthma & $27295(11.7)$ & $30586(13.0)$ & $<0.001$ & 22204 (13.2) & $6705(12.6)$ & 1677 (12.4) \\
\hline COPD & $14520(6.2)$ & $17740(7.6)$ & $<0.001$ & $12019(7.2)$ & $4554(8.6)$ & $1167(8.6)$ \\
\hline Diabetes mellitus & $23494(10.1)$ & $51442(21.9)$ & $<0.001$ & 35699 (21.2) & $12145(22.8)$ & 3598 (26.5) \\
\hline Severe mental illness & $2808(1.2)$ & $4281(1.8)$ & $<0.001$ & $3070(1.8)$ & $950(1.8)$ & $261(1.9)$ \\
\hline Epilepsy & $3596(1.5)$ & $3459(1.5)$ & 0.053 & $2456(1.5)$ & $787(1.5)$ & $216(1.6)$ \\
\hline Depression & 36309 (15.6) & $44243(18.8)$ & $<0.001$ & 32224 (19.2) & 9718 (18.2) & $2301(17.0)$ \\
\hline Coronary artery disease & $26875(11.5)$ & $52603(22.4)$ & $<0.001$ & $35485(21.1)$ & $13471(25.3)$ & 3647 (26.9) \\
\hline Heart failure & $7151(3.1)$ & $22976(9.8)$ & $<0.001$ & $12167(7.2)$ & $7887(14.8)$ & $2922(21.5)$ \\
\hline $\begin{array}{l}\text { Peripheral arterial } \\
\text { disease }\end{array}$ & $7236(3.1)$ & $14440(6.1)$ & $<0.001$ & $8950(5.3)$ & $4191(7.9)$ & $1299(9.6)$ \\
\hline Stroke or TIA & 11017 (4.7) & 18665 (7.9) & $<0.001$ & 12075 (7.2) & 5095 (9.6) & 1495 (11.0) \\
\hline Atrial fibrillation & $14556(6.2)$ & $28345(12.1)$ & $<0.001$ & $18411(11.0)$ & 7908 (14.8) & $2026(14.9)$ \\
\hline Hypertension & $123843(53.1)$ & 198199 (84.3) & $<0.001$ & $137848(82.0)$ & $47839(89.8)$ & $12512(92.2)$ \\
\hline Rheumatoid arthritis & $4129(1.8)$ & $5874(2.5)$ & $<0.001$ & $4139(2.5)$ & $1386(2.6)$ & $349(2.6)$ \\
\hline Osteoporosis & $15551(6.7)$ & $15873(6.8)$ & 0.211 & 11169 (6.6) & 3817 (7.2) & $887(6.5)$ \\
\hline
\end{tabular}




\begin{tabular}{|c|c|c|c|c|c|c|}
\hline \multirow[b]{3}{*}{ Variables } & \multicolumn{3}{|c|}{ By CKD status ( $n=468399)$} & \multicolumn{3}{|c|}{ By CKD stage $(n=235004)$} \\
\hline & $\begin{array}{l}\text { Matched } \\
\text { patients } \\
\text { without known } \\
\text { CKD } \\
(n=233395)\end{array}$ & $\begin{array}{l}\text { Matched } \\
\text { patients with } \\
\text { CKD } \\
(n=235004)\end{array}$ & $P$ value & $\begin{array}{l}\text { CKD stage 3a } \\
(n=168154)\end{array}$ & $\begin{array}{l}\text { CKD stage } 3 b \\
(n=53285)\end{array}$ & $\begin{array}{l}\text { CKD stage } 4 / 5 \\
(n=13565)\end{array}$ \\
\hline & $\mathbf{N}(\%)$ & $\mathbf{N}(\%)$ & & N (\%) & N (\%) & $\mathbf{N}(\%)$ \\
\hline Cancer & 45567 (19.5) & $52774(22.5)$ & $<0.001$ & 36339 (21.6) & $12939(24.3)$ & 3496 (25.8) \\
\hline
\end{tabular}

CKD, chronic kidney disease; COPD, chronic obstructive pulmonary disease; TIA, transient ischaemic attack.

\section{Comparison with previous studies}

Previous cohort studies reported that CKD may be independently associated with higher incident dementia or cognitive dysfunction. ${ }^{14}{ }^{36-38}$ According to a recent meta-analysis evaluating population-based prospective studies ${ }^{15}$ there is a marginally significant association between decreased kidney function (eGFR $<60 \mathrm{~mL}$ / $\min / 1.73 \mathrm{~m}^{2}$ ) and incidence of cognitive impairment or dementia, at a relative risk of 1.28 (95\% CI 0.99 to 1.65 ). This estimate overlapped with our estimate (fully adjusted RR of $1.12,95 \% 1.08$ to 1.16 ) of the association between CKD and dementia diagnosis after 6 months of cohort entry. Although there are some differences (eg, identification of dementia) between prospective cohort studies included in the meta-analysis and our study, based on routinely collected primary care data, a long-term weak association between CKD status and incident dementia appears to be present.

\section{Possible explanations of findings}

Dementia and CKD have shared risk factors; in particular, cardiovascular risk factors (such as smoking, obesity and diabetes mellitus) have been mainly considered to be a pathophysiological explanation affecting the kidneys and brain. ${ }^{6-10}$ Non-traditional CKD-specific risk factors including anaemia, electrolyte disorders (eg, hyponatraemia), poor nutrition and chronic inflammation (eg, oxidative stress and elevated inflammatory cytokines) are also considered to play a role in the brain-kidney relationship. ${ }^{6}$ In our study, there was a greater prevalence

Table 2 Incident rate ratio for dementia diagnosis and all-cause mortality comparing patients with and without CKD (stages $3-5)$ in the first 6 months of follow-up and thereafter

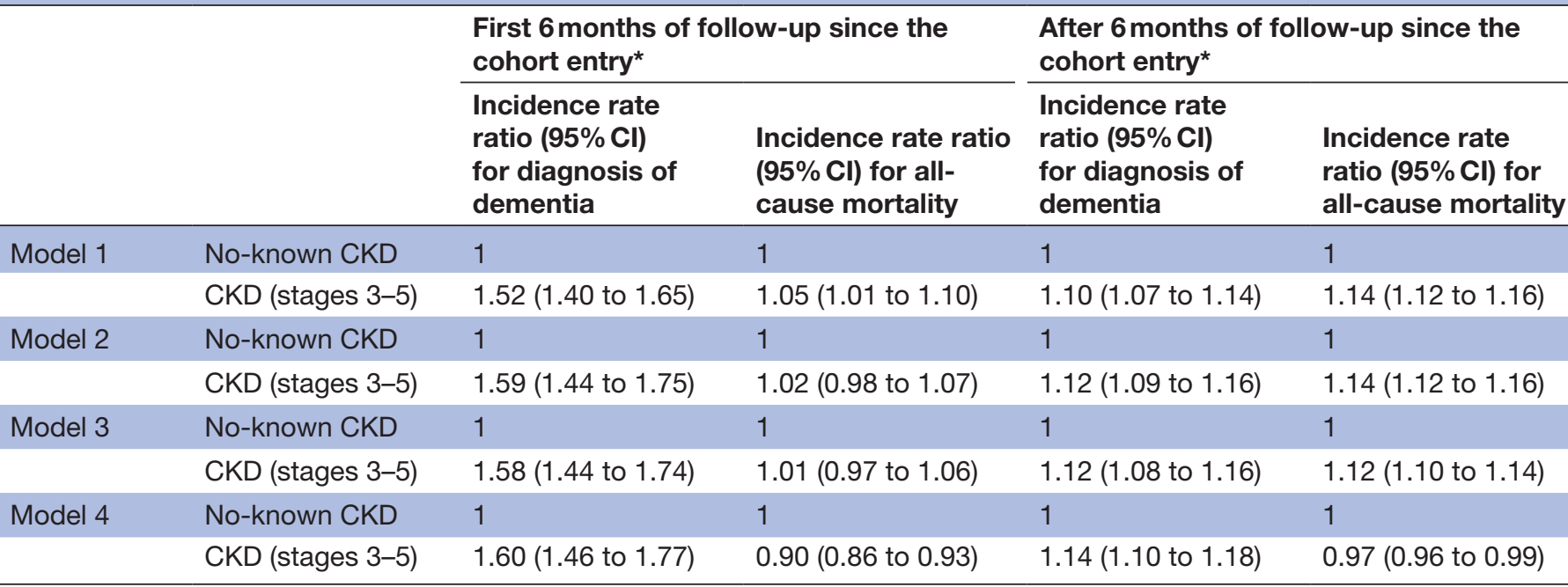

Model 1: adjusted for age, sex, general practice and calendar time.

Model 2: model 1+adjusted for ethnicity, socioeconomic status, smoking, body mass index and diabetes mellitus.

Model 3: model 2+adjusted for rheumatoid arthritis, chronic obstructive pulmonary disease, hypothyroidism, severe mental disease and depression.

Model 4: model 3+adjusted for hypertension and cardiovascular diseases (coronary artery disease, peripheral arterial disease, heart failure and stroke or transient ischaemic attack).

*Date when patients satisfied the CKD definition (ie, second estimated glomerular filtration rate $<60 \mathrm{~mL} / \mathrm{min} / 1.73 \mathrm{~m}^{2}$ ) and the same date for matched patients without known CKD.

CKD, chronic kidney disease. 
Table 3 Incident rate ratio for dementia diagnosis and all-cause mortality comparing patients with different stage of CKD (stages 3-5) in the first 6 months of follow-up and thereafter

\begin{tabular}{|c|c|c|c|c|c|}
\hline & & \multicolumn{2}{|c|}{$\begin{array}{l}\text { First } 6 \text { months of follow-up since the cohort } \\
\text { entry* }\end{array}$} & \multicolumn{2}{|c|}{$\begin{array}{l}\text { After } 6 \text { months of follow-up since the } \\
\text { cohort entry* }\end{array}$} \\
\hline & & $\begin{array}{l}\text { Incidence rate } \\
\text { ratio }(95 \% \mathrm{Cl}) \\
\text { for diagnosis of } \\
\text { dementia }\end{array}$ & $\begin{array}{l}\text { Incidence rate ratio } \\
(95 \% \mathrm{Cl}) \text { for all-cause } \\
\text { mortality }\end{array}$ & $\begin{array}{l}\text { Incidence rate } \\
\text { ratio }(95 \% \mathrm{Cl}) \\
\text { for diagnosis of } \\
\text { dementia }\end{array}$ & $\begin{array}{l}\text { Incidence rate } \\
\text { ratio }(95 \% \mathrm{Cl}) \text { for } \\
\text { all-cause mortality }\end{array}$ \\
\hline \multirow[t]{2}{*}{ Model 1} & CKD stage $3 a$ & 1 & 1 & 1 & 1 \\
\hline & CKD stage $4 / 5$ & 1.07 (0.87 to 1.30$)$ & 4.39 (4.15 to 4.65$)$ & 0.96 (0.91 to 1.00$)$ & 2.61 (2.54 to 2.70 ) \\
\hline \multirow[t]{3}{*}{ Model 2} & CKD stage $3 a$ & 1 & 1 & 1 & 1 \\
\hline & CKD stage $3 b$ & 1.04 (0.91 to 1.18$)$ & 1.85 (1.75 to 1.97$)$ & 0.97 (0.92 to 1.01) & 1.51 (1.48 to 1.55$)$ \\
\hline & CKD stage $4 / 5$ & $0.94(0.74$ to 1.20$)$ & 4.36 (4.09 to 4.64$)$ & 0.89 (0.80 to 0.98$)$ & 2.57 (2.49 to 2.65 ) \\
\hline Model 3 & CKD stage 3a & 1 & 1 & 1 & 1 \\
\hline \multirow[t]{3}{*}{ Model 4} & CKD stage $3 a$ & 1 & 1 & 1 & 1 \\
\hline & CKD stage $3 b$ & 1.05 (0.92 to 1.20$)$ & 1.78 (1.68 to 1.89$)$ & 0.97 (0.93 to 1.03 ) & 1.47 (1.44 to 1.50$)$ \\
\hline & CKD stage $4 / 5$ & $0.85(0.75$ to 1.21$)$ & 4.07 (3.82 to 4.33$)$ & $0.89(0.81$ to 1.00$)$ & 2.41 (2.34 to 2.49$)$ \\
\hline
\end{tabular}

Model 1: adjusted for age, sex, general practice and calendar time.

Model 2: model 1+adjusted for ethnicity, socioeconomic status, smoking, body mass index and diabetes mellitus.

Model 3: model 2+adjusted for rheumatoid arthritis, chronic obstructive pulmonary disease, hypothyroidism, severe mental disease and depression.

Model 4: model 3+adjusted for hypertension and cardiovascular diseases (coronary artery disease, peripheral arterial disease, heart failure and stroke or transient ischaemic attack).

${ }^{*}$ Date when patients satisfied the CKD definition (ie, second estimated glomerular filtration rate $<60 \mathrm{~mL} / \mathrm{min} / 1.73 \mathrm{~m}^{2}$ ).

CKD, chronic kidney disease.

of cardiovascular risk factors in the CKD cohort than in the matched no-known CKD cohort. However, a statistical adjustment for these factors did not fully attenuate the association between CKD and dementia diagnosis, even in the long-term, suggesting that the non-traditional CKD-specific factors (not measured in the current study) may be (part of) reasons for the observed CKD-dementia association.

The current study highlights a higher relative risk for incident diagnosis of dementia among patients with CKD in the first 6 months, compared with $>6$ months since the identification of CKD (and the same date for matched patients without known CKD). Caution is needed to interpret this result: it is very unlikely that CKD itself, just by being detected, causes faster rate of cognitive decline. Instead, patients with CKD (based on blood tests) seem to get more medical attention (eg, more consultations, more tests) compared with patients without CKD, and therefore are more likely to be diagnosed with other conditions such as dementia. After removing this effect in the first 6 months, the remaining weak association is probably closer to the true association between CKD and dementia. In addition, a greater mortality was seen in advanced CKD stages, suggesting that patients with advanced CKD are more likely to die before they would be diagnosed with dementia (ie, competing mortality risk).

\section{Strengths and weaknesses}

The strengths of the current study include its large sample size, which allowed us to conduct a statistical adjustment for a wide range of comorbidities and subgroup analyses. In addition, the CPRD is representative of the general English population, and therefore the study result is likely to be generalisable to the wider country. ${ }^{16}$ Furthermore, our previous validation study suggested that the CPRD captures most people with decreased kidney function (eGFR $<60 \mathrm{~mL} / \mathrm{min} / 1.73 \mathrm{~m}^{2}$ ), compared with nationally representative statistics (Health Survey for England), based on blood test results (without using recorded diagnosis of CKD) ${ }^{23}$ In contrast to previous studies that have associated single-time point measures of eGFR with incident dementia, here, we used the clinical definition including the chronicity of CKD to investigate the association. In the statistical analyses, care was taken to investigate the statistical assumptions of the models that were fitted, thereby identifying the time dependency of dementia diagnosis in routine care.

However, we need to acknowledge several limitations. First, the study outcome was defined as dementia diagnosis recorded by primary care physicians. According to a previous study, ${ }^{24}{ }^{25}$ dementia diagnosis in UK primary care showed a highly positive predictive value, but its sensitivity remains unknown. It is very possible that the 
sensitivity of dementia diagnosis is low, compared with dementia diagnosis based on more objective tools such as the Mini Mental State Examination, which would result in an underestimation of the association between CKD and incident dementia. In addition, we were unable to classify the type of dementia (eg, vascular dementia and Alzheimer's disease) accurately in our datasets, although the associations between each type of dementia and CKD may be different, from the pathophysiological perspective. Second, although we used the Chronic Kidney Disease Epidemiology Collaboration equation to estimate people's eGFR from serum creatinine, this approach might have overestimated kidney function in individuals who have lower muscle mass, resulting in a misclassification of CKD status among frail individuals who are at risk of dementia, ${ }^{39}$ again leading to an underestimation of the true association. In addition, we acknowledge that proteinuria is an important prediction marker for dementia, ${ }^{9}$ and proteinuria is also part of (early stage) CKD definition. ${ }^{21}{ }^{35}$ However, we did not define (early stage) CKD based on proteinuria, stratify patients by level of proteinuria or quantify the association between proteinuria and incidence of dementia. This was because proteinuria was infrequently checked in the CPRD (unless patients had diabetes mellitus), ${ }^{40}$ and it would not be appropriate to assume that people who did not undergo urine testing did not have proteinuria. For this reason, our CKD cohort might have missed some patients with true CKD, while the matched comparison cohort (no-known CKD cohort) might have included some patients with CKD, possibly diluting the observed association between CKD and dementia.

\section{Clinical and research implications}

As for clinical implications of the current study, co-occurrence of CKD and dementia in the primary care setting may mean that a subset of people with CKD are detected with having dementia, as they struggle coping with the multimorbidity that is frequently found in CKD. This study and earlier work showing the extent of mild cognitive impairment in early $\mathrm{CKD}^{41}$ suggest that the current system of a brief primary care appointment may not enable patients to manage their health problems due to the difficulty recalling complex information, which in turn may be an additional factor why people with CKD have poor health outcomes. A tailor-made consultation for high-risk patients may be needed.

As for research implications, our study highlights that a careful approach is needed to investigate a disease-disease association in routinely collected data such as the CPRD. We suggested that the true association between CKD and dementia would be much smaller than the apparent association estimated without considering potential biases (related to time since diagnosis, confounding and competing risk of mortality) inherent in the real-world data.

In addition, further research using traditional cohort data is needed to independently determine whether better management of risk factors present in people with CKD may diminish the impacts on cognitive impairment, and the observed long-term associations with dementia.

\section{CONCLUSION}

Using English primary care routine data, this study showed a co-occurrence of the detection of CKD and dementia in real-world clinical practice in the first 6 months after CKD detection, while a weak association was suggested in the long run. There was no positive association between CKD severity and dementia diagnosis, most likely due to competing mortality.

Acknowledgements The authors would like to thank the patients and clinicians who have contributed data to the CPRD to enable this study.

Contributors $\mathrm{RH}$ and DN planned the study. MI carried out the data extraction and conducted the data processing. RH analysed the data, interpreted the results and drafted the manuscript. DN and MI contributed substantially to the study design, interpretation of the results and writing of the manuscript. All authors read and approved the final manuscript.

Funding The authors have not declared a specific grant for this research from any funding agency in the public, commercial or not-for-profit sectors.

Competing interests None declared.

Patient and public involvement Patients and/or the public were not involved in the design, conduct, reporting or dissemination plans of this research.

Patient consent for publication Obtained.

Ethics approval We obtained study approval from the institutional review board of the London School of Hygiene and Tropical Medicine (reference: 13943), and the CPRD's Independent Scientific Advisory Committee (Protocol: 17_123R).

Provenance and peer review Not commissioned; externally peer reviewed.

Data availability statement Data may be obtained from a third party and are not publicly available. The data were obtained from the Clinical Practice Research Datalink (CPRD). CPRD data governance does not allow us to distribute patient data to other parties. Researchers may apply for data access at http://www.CPRD.com/.

Open access This is an open access article distributed in accordance with the Creative Commons Attribution Non Commercial (CC BY-NC 4.0) license, which permits others to distribute, remix, adapt, build upon this work non-commercially, and license their derivative works on different terms, provided the original work is properly cited, appropriate credit is given, any changes made indicated, and the use is non-commercial. See: http://creativecommons.org/licenses/by-nc/4.0/.

ORCID iD

Masao Iwagami http://orcid.org/0000-0001-7079-0640

\section{REFERENCES}

1 GBD 2015 DALYs and HALE Collaborators. Global, regional, and national disability-adjusted life-years (DALYs) for 315 diseases and injuries and healthy life expectancy (HALE), 1990-2015: a systematic analysis for the global burden of disease study 2015. Lancet 2016;388:1603-58.

2 GBD 2016 Dementia Collaborators. Global, regional, and national burden of Alzheimer's disease and other dementias, 1990-2016: a systematic analysis for the global burden of disease study 2016. Lancet Neurol 2019;18:88-106.

3 Coresh J. Update on the burden of CKD. J Am Soc Nephrol 2017;28:1020-2.

4 Chen L, Reed C, Happich M, et al. Health care resource utilisation in primary care prior to and after a diagnosis of Alzheimer's disease: a retrospective, matched case-control study in the United Kingdom. BMC Geriatr 2014;14:76.

5 Kent S, Schlackow I, Lozano-Kühne J, et al. What is the impact of chronic kidney disease stage and cardiovascular disease on the annual cost of hospital care in moderate-to-severe kidney disease? BMC Nephrol 2015;16:65. 
6 Drew DA, Weiner DE, Sarnak MJ. Cognitive impairment in CKD: pathophysiology, management, and prevention. Am J Kidney Dis 2019;74:782-90.

7 Zammit AR, Katz MJ, Bitzer M, et al. Cognitive impairment and dementia in older adults with chronic kidney disease: a review. Alzheimer Dis Assoc Disord 2016;30:357-66.

8 Viggiano D, Wagner CA, Blankestijn PJ, et al. Mild cognitive impairment and kidney disease: clinical aspects. Nephrol Dial Transplant 2020;35:10-17.

9 Bugnicourt J-M, Godefroy O, Chillon J-M, et al. Cognitive disorders and dementia in CKD: the neglected kidney-brain axis. J Am Soc Nephrol 2013;24:353-63.

10 Elias MF, Dore GA, Davey A. Kidney disease and cognitive function. Contrib Nephrol 2013;179:42-57.

11 Mogi M, Horiuchi M. Clinical interaction between brain and kidney in small vessel disease. Cardiol Res Pract 2011;2011:1-5.

12 Kurella M, Chertow GM, Fried LF, et al. Chronic kidney disease and cognitive impairment in the elderly: the health, aging, and body composition study. J Am Soc Nephrol 2005;16:2127-33.

13 Yaffe K, Ackerson L, Kurella Tamura M, et al. Chronic kidney disease and cognitive function in older adults: findings from the chronic renal insufficiency cohort cognitive study. J Am Geriatr Soc 2010;58:338-45.

14 Miwa K, Tanaka M, Okazaki S, et al. Chronic kidney disease is associated with dementia independent of cerebral small-vessel disease. Neurology 2014;82:1051-7.

15 Deckers K, Camerino I, van Boxtel MPJ, et al. Dementia risk in renal dysfunction: a systematic review and meta-analysis of prospective studies. Neurology 2017;88:198-208.

16 Herrett E, Gallagher AM, Bhaskaran K, et al. Data resource profile: clinical practice research Datalink (CPRD). Int J Epidemiol 2015;44:827-36.

17 Mathur R, Bhaskaran K, Chaturvedi N, et al. Completeness and usability of ethnicity data in UK-based primary care and hospital databases. J Public Health 2014;36:684-92.

18 Department for Communities and Local Government. English indices of deprivation, 2010. Available: https://www.gov.uk/government/ statistics/english-indices-of-deprivation-2010 [Accessed 1 Jun 2019].

19 Lewis JD, Bilker WB, Weinstein RB, et al. The relationship between time since registration and measured incidence rates in the general practice research database. Pharmacoepidemiol Drug Saf 2005;14:443-51.

20 McDonald HI, Shaw C, Thomas SL, et al. Methodological challenges when carrying out research on CKD and AKI using routine electronic health records. Kidney Int 2016;90:943-9.

21 National Kidney Foundation. K/DOQI clinical practice guidelines for chronic kidney disease: evaluation, classification, and stratification. Am J Kidney Dis 2002;39:S1-266.

22 Levey AS, Stevens LA, Schmid $\mathrm{CH}$, et al. A new equation to estimate glomerular filtration rate. Ann Intern Med 2009;150:604-12.

23 Iwagami M, Tomlinson LA, Mansfield KE, et al. Validity of estimated prevalence of decreased kidney function and renal replacement therapy from primary care electronic health records compared with national survey and registry data in the United Kingdom. Nephrol Dial Transplant 2017;32:ii142-50.
24 Seshadri S, Zornberg GL, Derby LE, et al. Postmenopausal estrogen replacement therapy and the risk of Alzheimer disease. Arch Neurol 2001;58:435-40.

25 Dunn N, Mullee M, Perry VH, et al. Association between dementia and infectious disease: evidence from a case-control study. Alzheimer Dis Assoc Disord 2005;19:91-4.

26 Hippisley-Cox J, Coupland C, Brindle P. Development and validation of QRISK3 risk prediction algorithms to estimate future risk of cardiovascular disease: prospective cohort study. BMJ 2017;357:j2099.

27 Kuo S-C, Lai S-W, Hung H-C, et al. Association between comorbidities and dementia in diabetes mellitus patients: populationbased retrospective cohort study. J Diabetes Complications 2015;29:1071-6.

28 Lin C-E, Chung C-H, Chen L-F, et al. Increased risk of dementia in patients with schizophrenia: a population-based cohort study in Taiwan. Eur Psychiatry 2018;53:7-16.

29 Hickson LJ, Crowson CS, Gabriel SE, et al. Development of reduced kidney function in rheumatoid arthritis. Am J Kidney Dis 2014;63:206-13.

30 Petersen LE, Baptista TSA, Molina JK, et al. Cognitive impairment in rheumatoid arthritis: role of lymphocyte subsets, cytokines and neurotrophic factors. Clin Rheumatol 2018;37:1171-81.

31 Liao K-M, Ho C-H, Ko S-C, et al. Increased risk of dementia in patients with chronic obstructive pulmonary disease. Medicine 2015;94:e930.

32 Parsaik AK, Singh B, Roberts RO, et al. Hypothyroidism and risk of mild cognitive impairment in elderly persons: a population-based study. JAMA Neurol 2014;71:201-7.

33 Meuwese CL, van Diepen M, Cappola AR, et al. Low thyroid function is not associated with an accelerated deterioration in renal function. Nephrol Dial Transplant 2019;34:650-9.

34 Noordzij M, Leffondré K, van Stralen KJ, et al. When do we need competing risks methods for survival analysis in nephrology? Nephrol Dial Transplant 2013;28:2670-7.

35 Kidney Disease: Improving Global Outcomes (KDIGO). KDIGO 2012 clinical Practice Guideline for the evaluation and management of chronic kidney disease, 2013: 1-150.

36 Sasaki Y, Marioni R, Kasai M, et al. Chronic kidney disease: a risk factor for dementia onset: a population-based study. The Osaki-Tajiri project. J Am Geriatr Soc 2011;59:1175-81.

37 Etgen T, Sander D, Chonchol M, et al. Chronic kidney disease is associated with incident cognitive impairment in the elderly: the invade study. Nephrol Dial Transplant 2009;24:3144-50.

38 Feng L, Yap KB, Yeoh LY, et al. Kidney function and cognitive and functional decline in elderly adults: findings from the Singapore longitudinal aging study. J Am Geriatr Soc 2012;60:1208-14.

39 Kulmala J, Nykänen I, Mänty M, et al. Association between frailty and dementia: a population-based study. Gerontology 2014;60:16-21.

40 Health Quality Improvement Partnership. National chronic kidney disease audit: national report (Part 1), 2017. Available: https:// www.hqip.org.uk/resources/national-chronic-kidney-disease-auditnational-report-part-1/ [Accessed 1 Jun 2019].

41 Silverwood RJ, Richards M, Pierce M, et al. Cognitive and kidney function: results from a British birth cohort reaching retirement age. PLoS One 2014;9:e86743. 